\title{
Comparação da gravidade dos casos de dengue segundo a classificação antiga e a classificação revisada
}

\author{
Comparison of dengue cases severity according to the dengue classification \\ and to the dengue revised classification
}

\section{Kleber Giovanni Luz¹, Ana Beatriz Seabra Santos de Araújo², Glauco Igor Viana dos Santos ${ }^{3}$, Luísa Silva de Sousa², Maria Beatriz Nóbrega Eberlin², Sâmea Costa Pinheiro Guerra², Yngra Bastos Mesquita Minora de Almeida ${ }^{2}$}

Luz KG, Araujo ABSS, Santos GIV, Sousa LS, Eberlin MBN, Guerra SCP, Almeida YBMM. Comparação da gravidade dos casos de dengue segundo a classificação antiga e a classificação revisada / Comparison of severity of dengue cases according to the dengue classification and the dengue revised classification. Rev Med (São Paulo). 2018 nov.-dec.;97(6):547-53.

RESUMO: Introdução: Existem duas classificações que estratificam os casos de dengue pelo quadro clínico laboratorial: a classificação proposta nos anos 50 e a revisada pela Organização Mundial de Saúde, adotada em janeiro de 2014 no Brasil. Compará-las quanto à capacidade de identificar a gravidade do caso representa nosso objetivo. Métodos: Estudo observacional e transversal com análise e comparação dos casos de dengue de 2011 a 2013 de um hospital terciário de referência da cidade de Natal/RN, de acordo com a classificação antiga e a classificação revisada. As correspondências adotadas foram: Dengue Clássica e Febre Hemorrágica da Dengue (DHF) grau I com Dengue; DHF grau II com Dengue com sinais de alarme; DHF grau III e IV com Dengue grave. Resultados: 2.318 fichas foram analisadas, com a população predominantemente adulta, média de idade 30,32 anos $\pm 17,89$, sendo $39 \%$ do sexo masculino, $61 \%$ do sexo feminino. A partir das correlações designadas, 428 casos foram concordantes, 699 discordantes e 1191 "sem classificação" (casos cujos dados dos prontuários não possibilitaram sua classificação). Conclusões: As duas classificações foram equivalentes no manejo clínico quando os casos de dengue foram graves. A classificação antiga evita a superestimação de casos leves e moderados por utilizar mais aspectos clínicos e laboratoriais que a classificação revisada. Sangramento de mucosa, dor abdominal e vômitos não representaram sinais que evoluíram para gravidade, demonstrando como a utilização dos sinais de alarme de maneira imprecisa pode superestimar os dados.

Descritores: Dengue; Dengue/classificação; Infecções por arbovirus; Classificação; Dengue grave.

ABSTRACT: Introduction: two classifications stratify cases of dengue according to clinical and laboratory findings: the classification proposed in the $50 \mathrm{~s}$ and the classification revised by the World Health Organization (WHO), which has been adopted in Brazil since January 2014. Our objective was to compare the two classification methods regarding their capability of identifying the severity of each case. Methods: Cross-sectional observational study with analysis and comparisons of dengue cases which occurred from 2011 to 2013 in a tertiary referral hospital in the city of Natal/RN, Brazil, according to the Dengue Classification and the Revised Dengue Classification. The equivalence adopted was: Classic Dengue and Dengue Hemorrhagic Fever (DHF) grade I

1.Universidade Federal do Rio Grande do Norte (UFRN), Hospital Giselda Trigueiro (HGT). Médico Infectologista com residência médica no Instituto de Infectologia Emílio Ribas -SP, Mestre em Pediatria pela UNIFESP e Doutor em Moléstias Infecciosas pela USP-SP. ORCID: https://orcid.org/0000-0003-3025-0660.E-mail: klebergluz@gmail.com

2.Universidade Federal do Rio Grande do Norte. Médica(o) generalista pela UFRN. ORCID: Araujo ABS - https://orcid.org/0000-00019868-9894; Sousa LS - ORCID: https://orcid.org/0000-0001-7467-5025; Eberlin MBN - https://orcid.org/0000-0003-4851-154X; Guerra SCP - https://orcid.org/0000-0002-1987-298X; Almeida YBMM - https://orcid.org/0000-0002-7781-8869. E-mail: anabeatrizssa@ gmail.com, luisasilvasousa@hotmail.com,mbianobrega@gmail.com,sameacosta@gmail.com. yngrabastos@gmail.com.

3.Universidade Federal do Rio Grande do Norte, Hospital Giselda Trigueiro. ORCID: https://orcid.org/0000-0001-7309-6806. Médico Infectologista com residência médica na Universidade Federal do Rio Grande do Norte. E-mail: glaucoviana_@hotmail.com.

Endereco para correspondência: Luísa Silva de Sousa. Rua Pinto Martins, 954. Areia Preta, Natal, RN. Brasil. CEP: 59014-060.

E-mail: luisasilvasousa@hotmail.com. 
Luz KG, et al. Comparação da gravidade dos casos de dengue segundo a classificação.

with Dengue; DHF grade II with Dengue with warning signs and DHF III and IV with Severe Dengue. Results: 2,318 records were analyzed, with a mean age of 30.32 years \pm 17.89 , and a population $39 \%$ male and $61 \%$ female. Based on the designated equivalence, 428 cases were concordant, 699 were discordant (212 classified as Classic Dengue and Dengue with warning signs - mucosal bleed, 62 as Classic Dengue and Dengue with warning signs abdominal pain) and 1,191 "without classification" (cases whose medical records did not allow classification). Conclusion: The two classifications were equivalent in clinical management when cases

\section{INTRODUÇÃO}

Dengue é a arbovirose mais importante na atualidade por sua significância em termos de morbidade e mortalidade. O número de casos de Dengue no Brasil teve crescimento significativo, passando de 40.179 em 1990 para 1.500 .535 em $2016^{1}$. Nos últimos dez anos, as epidemias de dengue aumentaram devido ao processo de urbanização, cujo caráter desorganizado gera uma infraestrutura deficitária das cidades que dificulta os métodos tradicionais de controle do vetor: o Aedes ${ }^{2}$.

No estado do Rio Grande do Norte (RN), no ano de 2016, o índice de infestação pelo mosquito A. aegypti apresentou uma abrangência de $80,84 \%$ dos municípios do estado. Dos casos suspeitos de Dengue, houve uma incidência acumulada de 1.844,24/100.000 habitantes, valor substancialmente mais elevado que o ano anterior, com incidência de 815,59/100.000 habitantes ${ }^{3}$.

Na história do estudo dos casos de Dengue, suas diferentes manifestações clínicas foram inicialmente agrupadas pela World Health Organization (WHO) / Organização Mundial da Saúde (OMS), desde os anos 70, nos seguintes espectros clínicos: a forma assintomática, na qual a presença do vírus no organismo é clinicamente inaparente; a febre da Dengue Clássica; a Febre Hemorrágica da Dengue (FHD) e a Síndrome de Choque da Dengue (SCD) $)^{4,5,6}$.

A FHD e SCD iniciam de forma semelhante a dengue clássica, mas, na FHD, fenômenos hemorrágicos (espontâneos ou provocados) surgem entre o $2^{\circ}$ e $3^{\circ}$ dia de doença, além de alterações laboratoriais, como a plaquetopenia, elevação do hematócrito em $20 \%$ do basal, acúmulo de líquido em cavidades serosas, tais como derrame pleural, ascite e derrame pericárdico, e hipoalbuminemia. O quadro pode evoluir para choque (SCD), com sintomas secundários a um estado de insuficiência circulatória. É suficiente para detectar esse estágio a presença de pulso rápido e fino, pressão de pulso estreita (quando há uma diferença menor ou igual a 20 mmHg entre a pressão sistólica e a diastólica), sinais de instabilidade hemodinâmica: taquicardia, extremidades frias e enchimento capilar lento ou hipotensão. A FHD e a SCD são organizadas em quatro graus de gravidade: grau I, tem como manifestação hemorrágica a prova do laço positiva; já no grau II, o sangramento é espontâneo: epistaxe, gengivorragia, metrorragia, petéquias, hematúria, were severe. The old classification avoids an overestimation of mild and moderate cases by using more clinical and laboratory aspects than the new classification. Mucosal bleed, abdominal pain and vomiting did not represent signs that evolved to severity, demonstrating how the imprecise use of warning signs can overestimate the data.

Keywords: Dengue; Dengue/classification; Arbovirus infections; Classification; Severe dengue.

sangramento gastrointestinal e hemoptoicos. No grau III prevalecem os sinais de falha cardiocirculatória (pele fria e úmida, agitação, pulso rápido e fino, hipotensão postural, convergência das pressões sistólica e diastólica, hipotensão arterial evidente). O grau IV corresponde ao choque descompensado ${ }^{7}$.

Há também as formas clínicas atípicas da Dengue que trazem danos orgânicos como a insuficiência renal aguda, encefalopatia, miocardiopatia ou hepatopatia por dengue ${ }^{8}$.

Estudos com a classificação antiga da Dengue pela OMS demonstraram uma sensibilidade de $62 \%$ na detecção da síndrome do choque da Dengue e especificidade de $92 \%$ nos casos que precisaram de intervenção. ${ }^{9}$ Esses resultados e o de outros estudos geraram questionamentos sobre a acurácia dessa estratificação, em especial na detecção dos casos graves de dengue ${ }^{9,10,11}$.

Em 2009, a OMS publicou a classificação revisada da Dengue, com divisão dos casos em: dengue, dengue com sinais de alarme e dengue grave ${ }^{12}$. O Brasil adotou essa classificação em 2014, usando-a como guia para manejo clínico da doença pelos profissionais da saúde ${ }^{13}$.

O caso suspeito de dengue corresponde a indivíduos que vivam ou tenham viajado nos últimos quatorze dias para área onde esteja ocorrendo transmissão de dengue ou tenha a presença de Aedes aegypti, apresentem febre, usualmente entre dois e sete dias, e duas ou mais das seguintes manifestações: náuseas, vômitos, exantema, mialgias, artralgias, cefaleia, dor retro-orbital, petéquias ou prova do laço positiva e leucopenia. Já o caso suspeito de dengue com sinais de alarme é todo caso de dengue que, no período de defervescência da febre apresenta determinados sinais ou sintomas que são considerados de alarme. $\mathrm{O}$ caso suspeito de dengue grave é todo caso de dengue que apresenta um ou mais dos seguintes resultados: choque, pressão arterial convergente $\leq 20 \mathrm{mmHg}$; hipotensão arterial em fase tardia; acúmulo de líquidos com insuficiência respiratória; sangramento grave, segundo a avaliação do médico ou comprometimento grave de órgãos ${ }^{12,13}$.

A confirmação do caso suspeito de dengue deve ser pela demonstração laboratorial: sorologia IgM, NS1 teste rápido ou ELISA, isolamento viral, reação em cadeia da polimerase - PCR, imunohistoquimica. Será descartado todo caso suspeito de dengue que possui um ou mais dos seguintes critérios: diagnóstico laboratorial negativo (deve-se confirmar se as amostras foram coletadas no 
período adequado); não tenha critério de vínculo clínicoepidemiológico; tenha diagnóstico laboratorial de outra entidade clínica; seja um caso sem exame laboratorial, cujas investigações clínica e epidemiológica são compatíveis com outras doenças ${ }^{12,13}$.

A ênfase aos sinais de alarme na classificação revisada da OMS (2009) demonstra uma tentativa de aprimorar a acurácia da estratificação dos casos de dengue, em especial nas apresentações mais graves da doença ${ }^{14}$. Mas, compará-la com a classificação antiga, faz-se necessário para comprovar qual detém maior acurácia. Destarte, o presente estudo, comparou essas duas classificações sobre a capacidade de cada uma em identificar e estratificar os casos de dengue em casos leves, moderados e graves da doença.

\section{MÉTODOS}

Trata-se de um estudo observacional com delineamento transversal sobre a análise e comparação dos casos de dengue, de acordo com a classificação antiga e a classificação revisada.

O objeto de estudo foi a ficha de papel de notificação de Dengue do Sistema de Informação de Agravos de Notificação (SINAN), arquivadas no Núcleo de Epidemiologia do Hospital Giselda Trigueiro (HGT). Foram incluídas no estudo as fichas com caso notificado como suspeito de dengue (CID 10: A 90.0 e A 90.1) entre os anos de 2011, 2012 e 2013 e cujas informações foram suficientes para categorizar o caso nas duas classificações.

As fichas que não possuíam todas as informações necessárias para classificação do caso, foram denominadas "sem classificação" e excluídas do cálculo do Kappa. Obteve-se uma população estudada predominantemente adulta, com uma média de idade de 30,32 anos $\pm 17,89$, e do sexo feminino 687 (61\%), comparado a 440 (39\%) indivíduos do sexo masculino.

Os dados utilizados para as classificações foram os sinais clínicos, sintomas, exames complementares e as observações obtidas dos prontuários em cada caso explicitado nas fichas, tornando o estudo qualiquantitativo. Além disso, foram extraídas as seguintes informações epidemiológicas: número da ficha de notificação, idade, sexo, município de residência, data dos primeiros sintomas e data da internação.

A coleta dos dados foi realizada no período de julho de 2014 a janeiro de 2015, nas dependências do Núcleo de Epidemiologia do HGT. O preparo para coleta de dados enfatizou o estudo das duas classificações e as adaptações dos critérios das classificações explicitadas a seguir. Os cem primeiros questionários coletados, considerados como um estudo piloto, foram analisados para correção de incompatibilidades e determinação de melhorias na obtenção dos dados.

Nesse estudo, para ser considerado Dengue clássica dentro da classificação antiga, foram incluídos os indivíduos com síndrome febril que não apresentavam os seguintes critérios da Febre Hemorrágica da Dengue (FHD): plaquetas abaixo de $100.000 / \mathrm{mm}^{3}$; presença de manifestações hemorrágicas (espontâneas ou prova do laço positiva); sinal de extravasamento plasmático (hemoconcentração - aumento em $20 \%$ do menor hematócrito registrado ou uma relação hematócrito/ hemoglobina maior ou igual a 3,2; derrames cavitários e/ ou hipoproteinemia).

As estratificações na classificação revisada seguiram as orientações da OMS sobre os conceitos de caso suspeito de dengue, dengue com sinais de alarme e dengue grave. Alguns critérios para Dengue com sinais de alarme foram adaptados por não existirem especificações nas fichas de notificação. A presença de dor abdominal foi considerada como dor abdominal intensa e contínua; vômitos como vômitos persistentes; hemoptoicos foram inseridos no sangramento de mucosas, enquanto hematúria não foi incluída; hepatomegalia evidenciada dentro dos sinais e sintomas ou por ultrassonografia de abdômen, que não foram concomitantes com doenças hepatotrópicas, foi considerada independentemente de uma medição. Isso mostrou-se necessário, pois as informações nas fichas não eram suficientes para especificar esses sinais e sintomas.

Nos casos com sangramentos, o instrumento de coleta dos dados não fornece a avaliação de gravidade, de modo que a metrorragia foi considerada como sangramento de mucosa- sinal de alarme e não como sangramento grave.

A determinação da relação entre os estágios das classificações foi estabelecida pelos pesquisadores com a supervisão do orientador. As correspondências entre a classificação antiga e a classificação revisada foram: Dengue clássica e FHD grau I com Dengue; FHD grau II com Dengue com sinais de alarme; FHD grau III e IV com Dengue grave.

Os resultados foram organizados no Excel com os dados epidemiológicos, a classificação antiga, a nova e a comparação das classificações - concordantes ou discordantes. Comparações com resultado envolvendo menos de 6 casos não foram analisadas, por terem pequena expressividade diante da população total estudada.

\section{RESULTADOS}

Dentro do censo de casos suspeitos de Dengue entre 2011 e 2013, atendidos no HGT, obteve-se um quantitativo total das fichas de notificação de Dengue do SINAN igual a 2.318 (Tabela 1). Entre essas, 1191 (51,35\%) não se adequaram ao critério de inclusão no estudo, sendo denominadas fichas "sem classificação" e excluídas do cálculo do Kappa, 428 (18,5\%) casos concordaram quanto as correspondências das classificações e 699 (30,15\%) foram discordantes. Obteve-se uma população estudada 
Luz KG, et al. Comparação da gravidade dos casos de dengue segundo a classificação.

predominantemente adulta, com uma média de idade de 30,32 anos $\pm 17,89$, e do sexo feminino 687 (61\%), comparado a $440(39 \%)$ indivíduos do sexo masculino.

Os casos discordantes foram organizados comparando a classificação antiga com a classificação revisada e especificando o motivo da divergência (Tabela 2). Discordâncias inferiores a seis casos foram consideradas não significativas para análise.

Tabela 1 - Número de fichas analisadas de acordo com equivalência classificatória/ano

\begin{tabular}{|ccccc|}
\hline Ano & Concordante & Discordante & Sem classificação & Total analisado \\
\hline 2011 & 207 & 248 & 611 & 1066 \\
\hline 2012 & 162 & 342 & 542 & 1046 \\
\hline 2013 & 59 & 109 & 38 & 206 \\
\hline Total & 428 & 699 & 1191 & 2318 \\
\hline
\end{tabular}

Tabela 2 - Número total de fichas correspondentes às classificações discordantes em 2011, 2012 e 2013

\begin{tabular}{|c|c|c|c|}
\hline Classificação Antiga & Classificação Revisada & $\begin{array}{l}\text { Total } \\
\text { (n) }\end{array}$ & $\begin{array}{l}\text { Total } \\
(\%)\end{array}$ \\
\hline Dengue Clássica & Dengue com sinal de alarme (sangramento de mucosa) & 212 & 30,3 \\
\hline Dengue Clássica & Dengue com sinal de alarme (dor abdominal e vômitos) & 64 & 9,2 \\
\hline Dengue Clássica & Dengue com sinal de alarme (dor abdominal) & 62 & 8,9 \\
\hline Dengue Clássica & Dengue com sinal de alarme (vômitos) & 57 & 8,2 \\
\hline Dengue Clássica & $\begin{array}{l}\text { Dengue com sinal de alarme (dor abdominal e sangramento } \\
\text { de mucosa) }\end{array}$ & 48 & 6,9 \\
\hline Dengue Clássica & Dengue grave (pressão arterial convergente) & 37 & 5,3 \\
\hline Dengue Clássica & $\begin{array}{l}\text { Dengue com sinais de alarme (sangramento de mucosa e } \\
\text { vômitos) }\end{array}$ & 31 & 4,4 \\
\hline Dengue Clássica & $\begin{array}{l}\text { Dengue com sinais de alarme (dor abdominal, vômitos e } \\
\text { sangramento de mucosa) }\end{array}$ & 24 & 3,4 \\
\hline Dengue Clássica & Dengue grave (melena) & 19 & 2,7 \\
\hline FHD grau II & Dengue & 15 & 2,1 \\
\hline FHD grau II & Dengue grave (hematêmese e melena) & 15 & 2,1 \\
\hline FHD grau II & Dengue grave (hematêmese) & 11 & 1,6 \\
\hline FHD grau II & Dengue grave (melena) & 10 & 1,4 \\
\hline Dengue Clássica & Dengue grave (hipotensão) & 9 & 1,3 \\
\hline Dengue Clássica & Dengue grave (hipotensão e pressão arterial convergente) & 9 & 1,3 \\
\hline Dengue Clássica & Dengue com sinais de alarme (lipotímia) & 8 & 1,1 \\
\hline Dengue Clássica & Dengue grave (alteração do nível da consciência) & 6 & 0,86 \\
\hline Somatório das discordâncias não significativas* & & 62 & 8,9 \\
\hline
\end{tabular}

*Foram consideradas discordâncias não significativas aquelas em quantidade inferior a seis.

Os casos concordantes foram especificados (Tabela 3) e, de acordo com a correlação das classificações proposta neste estudo, a maior equivalência foi nos casos com sangramentos espontâneos e outros sinais de alarme: FHD grau II e Dengue com sinais de alarme (44\%).

Tabela 3 - Número de fichas correspondentes às classificações concordantes em 2011, 2012 e 2013.

\begin{tabular}{|llcc|}
\hline Classificação Antiga & Classificação Revisada & Total (n) & Total (\%) \\
\hline Dengue Clássica & Dengue & 163 & 38,1 \\
\hline FHD grau I & Dengue & 5 & 1,2 \\
\hline FHD grau II & Dengue com sinais de alarme & 189 & 44,1 \\
\hline FHD grau III & Dengue Grave & 65 & 15,2 \\
\hline FHD grau IV & Dengue Grave (Óbito) & 6 & 1,4 \\
\hline
\end{tabular}




\section{DISCUSSÃO}

Esse estudo pioneiro no RN teve como objetivo comparar a classificação antiga e a classificação revisada quanto à eficácia na capacidade de estratificação dos casos de dengue em leves, moderados e graves.

Os casos considerados graves foram aqueles que apresentaram uma evolução para SCD (com ou sem óbito). Dessa forma, representou um sinal de alarme importante para a estratificação adequada do caso, aquele o qual sugeriu uma progressão do quadro clínico para gravidade. O estudo multicêntrico DENCO encontrou o sangramento de mucosa como um sinal de alarme importante ${ }^{15,16}$. Entretanto no presente estudo, o sangramento de mucosa representou um sinal de alarme superestimado, pois 212 casos classificados como dengue clássica e dengue com sinal de alarme (nas classificações antiga e revisada, respectivamente) não evoluíram para SCD e/ou óbito.

Essa superestimação dos sinais de alarme poderia levar um maior número de pacientes a serem internados para conduta terapêutica precoce, a fim de evitar uma progressão da doença e piora do quadro. Conforme estudo realizado na Nicarágua ${ }^{17}$, esse aumento de internamentos não reflete fidedignamente a gravidade de todos os casos, demandando uma análise posterior do enfermo para definir a sua verdadeira condição. Isso pode gerar uma saturação do sistema de saúde com subsequente aumento dos custos necessários na saúde pública, em que casos de baixa ou moderada gravidade seriam manejados de forma discordante da real situação clínica. Possível explicação para esse fato seria a formatação da classificação revisada ser constituída apenas por critérios clínicos para a definição dos casos graves, enquanto que a antiga é acrescida de parâmetros laboratoriais para o diagnóstico.

Somado a isso, na discordância de 62 casos classificados como dengue clássica e dengue com sinal de alarme, devido à presença de dor abdominal, houve novamente uma superestimação do sinal de alarme, uma vez que nenhum desses indivíduos evoluiu para forma grave. Esse sintoma foi avaliado subjetivamente, visto que no modelo das fichas consultadas não havia referência à intensidade e temporalidade da dor. Ademais, para ser considerada sinal de alarme segundo à OMS, a dor abdominal deve ser intensa e contínua, mas não existem parâmetros específicos para quantificá-la, dificultando assim, a real estratificação do quadro pelo profissional de saúde.

Dessa forma, uma escala analógica da dor, capaz de determinar quantitativamente o grau do sintoma entre 0 e 10 (na qual 0 representa a não existência da dor e 10, a maior dor já sentida pelo paciente), poderia ser um instrumento prático e útil que auxiliaria ao doente quantificar a dor e o profissional da saúde a classificar o caso de forma mais fidedigna à realidade.

Com relação ao sintoma 'presença de vômitos', deveria ser considerado um sinal de alarme quando: os vômitos fossem persistentes com três ou mais episódios em uma hora ou houver cinco ou mais episódios em seis horas ${ }^{18}$. Assim, na análise dos dados, 57 indivíduos classificados como dengue clássica e como dengue com sinal de alarme, apresentaram vômitos como sinal de alarme registrado, contudo não evoluíram para gravidade. Esse sinal foi também superestimado, uma vez que não houve registro nas fichas da frequência e da quantidade de episódios de vômitos. Além disso, os vômitos poderiam ser causados pela própria doença ou por uso abusivo de medicações, o que dificultaria uma classificação realista.

Poder-se-ia imaginar que caso houvesse a presença concomitante de dois ou mais sinais de alarme mencionados acima em um mesmo paciente ( $\mathrm{n}=167$ ), significaria uma gravidade maior do quadro clínico. No entanto, não apresentaram essa evolução, mostrando mais uma vez que o sangramento de mucosa, a dor abdominal e a presença de vômitos seriam sinais de alarme superestimados.

De acordo com a classificação revisada, a pressão arterial convergente é sinal de choque. Nesse estudo, 37 casos de pressão arterial convergente foram classificados como dengue grave, mas não foram classificados como FHD pela classificação antiga. Possíveis motivos para essa discordância seriam: a falta de exames laboratoriais, a realização de um único exame ou a incapacidade de buscar o sangramento devido ao choque.

Situação semelhante aconteceu com os nove casos classificados como dengue clássica e dengue grave por hipotensão (pressão arterial menor que 90x60 mmHg), em que pode ter acontecido alteração hemodinâmica sem fuga capilar e isso é um aspecto importante, uma vez que todo paciente com hipotensão é grave. Nesse sentido, a classificação revisada favorece a presença de pressão arterial convergente como sinal de gravidade sem necessitar de sangramento.

Além disso, a espera por uma plaquetopenia e um hematócrito elevado pode não vir em tempo hábil para a definição do manejo nesses casos em específico. Essa questão coincide com uma provável limitação da classificação antiga em ter que aguardar os exames para estratificar o caso, perdendo um tempo que pode ser essencial à conduta adequada.

Os indivíduos $(\mathrm{n}=15)$ que apresentaram melena e hematêmese foram classificados como FHD grau II e dengue grave, ou seja, foram equivalentes quanto ao manejo inicial e conduta (internação), mas discordantes quanto às classificações.

Em seis casos, houve discordância de classificação entre dengue clássica e dengue grave devido à alteração do nível da consciência. A alteração do sensório pode representar desde um sangramento do sistema nervoso central (SNC) até uma encefalite, sendo um sinal de gravidade e, portanto, seria necessário que o indivíduo fosse manejado como grave. 
Luz KG, et al. Comparação da gravidade dos casos de dengue segundo a classificação.

As comparações nos resultados que envolveram menos de 6 casos não foram analisadas diante da menor expressividade frente à população estudada.

Uma vantagem desse estudo foi o fato da coleta de dados ter sido realizada a partir de uma fonte primária: a ficha do SINAN adaptada pelo Núcleo de Epidemiologia do HGT. Seu conteúdo inclui mais informações que o arquivo original, a exemplo da presença de observações adicionais que abrange importantes dados do paciente para a classificação do seu quadro clínico, como o valor da pressão arterial.

O estudo foi limitado aos casos atendidos no HGT podendo não ter representado o cenário do Estado do $\mathrm{RN}$ de forma geral, uma vez que o hospital é um centro de atenção terciária que atende predominantemente a população adulta da cidade de Natal. Somado a isso, essa população é predominantemente da etnia branca $(44,5 \%$ segundo IBGE 2010), podendo ter restringido as análises.

O princípio geral da classificação antiga em usar dados laboratoriais como critérios diagnósticos, comparado ao uso de dados clínicos na classificação revisada, evitaria a superestimação de casos leves e moderados.

Os resultados mostraram ainda que sangramento de mucosa, dor abdominal e vômito não representaram sinais que evoluíram para gravidade, demonstrando como a utilização dos sinais de alarme de maneira imprecisa pode superestimar os dados. Isso também foi demonstrado em um estudo multicêntrico realizado com 18 países $^{19}$, no qual foi percebido que ainda há uma necessidade de formação, divulgação e investigação adicional sobre os sinais de alarme. Somado a isso, um estudo europeu foi realizado objetivando comparar as mudanças entre as versões das duas classificações e reforçou que apesar da classificação revisada ser sensível ao diagnóstico de dengue grave, ainda há problemas com sua aplicabilidade. Isso porque a definição de sinais de alarme é muito ampla, exigindo uma mais específica ${ }^{20}$.

Por sua vez, as duas classificações apresentaram-se concordantes quanto ao manejo clínico quando os casos de dengue foram graves. Os pacientes classificados como FHD III e IV na classificação antiga e dengue grave na classificação revisada, conforme a Tabela 3, apresentaram necessidade de internação para cuidados intensivos visando uma monitorização hemodinâmica adequada com reposição volêmica eficaz e acesso contínuo aos exames laboratoriais necessários.

Assim, há uma necessidade do aprofundamento de estudos com comparações semelhantes e protocolos sistematizados para identificar a eficácia prática da classificação revisada comparada à classificação antiga.

Agradecimentos: Agradecemos aos pacientes e ao Hospital Giselda Trigueiro por serem nossa fonte de informações e local da coleta da nossa pesquisa.

Participação no artigo: Planejamento do projeto, processo de coleta e análise dos dados e a elaboração do manuscrito: Ana Beatriz Seabra, Luísa Silva, Maria Beatriz Nóbrega, Sâmea Costa, Yngra Bastos. Planejamento do projeto, orientação científica, revisor do manuscrito: Kleber Giovanni. Orientação científica, revisor do manuscrito e tradução para língua inglesa: Glauco Vianna.

\section{REFERÊNCIAS}

1. Brasil. Situação Epidemiológica/ Dados Dengue: Incidência e casos de Dengue Brasil, Grandes Regiões e Unidades Federadas de 1990 a 2016. Portal da Saúde. Disponível em: http://u.saude.gov.br/index.php/situacao-epidemiologicadados-dengue.

2. Zara ALSA, Santos SM, Fernandes-Oliveira ES, Carvalho RG, Coelho GE. Estratégias de controle do Aedes aegypti: uma revisão. Epidemiol Serv Saúde. 2016;25(2):391-404. doi: http://dx.doi.org/10.5123/s1679-49742016000200017.

3. Rio Grande do Norte. Governo do Estado. Boletim epidemiológico. Atualização da situação epidemiológica das arboviroses no Rio Grande do Norte. 52 ${ }^{\mathrm{a}}$ Semana Epidemiológica, 2016. Disponível em: http://adcon.rn.gov. br/ACERVO/sesap/DOC/DOC000000000140313.PDF.

4. Halstead SB. Observations related to pathogenesis of dengue hemorrhagic fever. VI. Hypotheses and discussion. Yale J Biol Med. 1970;42(5):350-62.

5. World Health Organization. Dengue haemorrhagic fever, diagnosis, treatment and control. Geneva; 1986.

6. World Health Organization. Dengue haemorrhagic fever: diagnosis, treatment, prevention and control. 2nd ed. Geneva; 1997.

7. Gubler DJ. Dengue and dengue hemorrhagic fever. Clin Microbiol Rev. 1998;11(3):480-96. doi: 10.1128/ CMR.11.3.480.

8. Nimmannitya S, Thisyakorn U, Hemsrichart V. Dengue haemorrhagic fever with unusual manifestations. Southeast Asian J Trop Med Public Health. 1987;18(3):398-406.

9. Srikiatkhachorn A, Gibbons RV, Green S, et al. Dengue hemorrhagic fever: the sensitivity and specificity of the World Health Organization definition for identification of severe cases of dengue in Thailand, 1994-2005. Clin Infect Dis. $2010 ; 50(8): 1135-43$. doi: 10.1086/651268.

10. Balmaseda A, Hammond SN, Perez MA, et al. Short report: assessment of the World Health Organization scheme for classification of dengue severity in Nicaragua. Am J Trop Med Hyg. 2005;73(6):1059-62. doi: https://doi.org/10.4269/ ajtmh.2005.73.1059.

11. Bandyopadhyay S, Lum LC, Kroeger A. Classifying dengue: a review of the difficulties in using the WHO case classification for dengue haemorrhagic fever. Trop Med Int Health. 2006;11(8):1238-55. doi: 10.1111/j.1365-3156.2006.01678.x. 
Luz KG, et al. Comparação da gravidade dos casos de dengue segundo a classificação.

12. World Health Organization. Dengue: guidelines for diagnosis, treatment, prevention and control. Geneva; 2009.

13. Brasil. Ministério da Saúde. Secretaria de Vigilância em Saúde. Dengue: diagnóstico e manejo clínico: adulto e criança - 5a ed. Brasília; 2016. Disponível em: http://bvsms.saude. gov.br/bvs/publicacoes/dengue_diagnostico_manejo_adulto_ crianca_3ed.pdf.

14. Verdeal JCR, et al. Recomendações para o manejo de pacientes com formas graves de dengue. Rev. bras. ter. intensiva. 2011;23(2):125-33. doi: http://dx.doi.org/10.1590/ S0103-507X2011000200004.

15. Alexander N, et al. Multicentre prospective study on dengue classification in four South-east Asian and three Latin American countries. Trop Med Int Health. 2011;16(8):936-48. doi: 10.1111/j.1365-3156.2011.02793.x.

16. Macedo GA, et al. Sensitivity and Specificity of the World Health Organization Dengue Classification Schemes for Severe Dengue Assessment in Children in Rio de Janeiro.
PLOS - Neglected Tropical Dise. 2014;9(4):e96314. doi: http://doi.org/10.1371/journal.pone.0096314.

17. Bandyopadhyay S, Lum LCS and Kroeger A. Classifying dengue: a review of the difficulties in using the WHO case classification for dengue haemorrhagic fever. Trop Med Int Health. 2006;11(8):1238-55. doi:10.1111/j.13653156.2006.01678.x

18. Torres EM. Dengue. Estud Avançados. 2008;22(64)33-52. doi: http://dx.doi.org/10.1590/S0103-40142008000300004.

19. Barniol J, Gaczkowski R, Barbato EV, et al. Usefulness and applicability of the revised dengue case classification by disease: multi-centre study in 18 countries. BMC Infect Dis. 2011;11:106. doi: 10.1186/1471-2334-11-106.

20. Hadinegoro SRS. The revised WHO dengue case classification: does the system need to be modified? Paediatr Int Child Health. 2012;32(s1):33-8. doi: http://doi.org/10.1179/20469 04712Z.00000000052.

Recebido: 27 dez. 2017.

Aceito: 07 jan. 2019. 